\section{¿Cómo es el mapa de jubilación en Argentina?}

\section{Enrique S. Dentice (h)}

El paso del tiempo nos lleva a enfrentar situaciones que nos parecen lejanas, pero un día nos llegan y la decisión es mezcla de incertidumbre y desconocimientos. Por eso, pensé en contar mi vivencia en un mapa, una hoja de ruta, para ir pensando. ${ }^{1}$

\section{SITUACIÓN OBJETIVA. LOS NÚMEROS}

El Gobierno argentino atravesó los primeros meses del año 2021 con un desequilibrio en las cuentas públicas muy inferior al de 2020.

Según el informe de la Fundación Mediterránea, que creó el Instituto de Estudios sobre la Realidad Argentina y Latinoamericana (IERAL), "el gasto previsional promedio de los primeros cinco meses de 2021, cayó 9,4\% en términos reales -es decir, considerando la inflación- con respecto de igual periodo de 2020 y en un $17,7 \%$ por debajo de igual periodo de $2018 " .{ }^{2}$

Las jubilaciones vienen arrastrando una pérdida de su poder adquisitivo desde mucho tiempo y la IERAL ${ }^{2}$ estimó que "la jubilación mínima cayó un $23,5 \%$ entre 2013 y 2021 ; mientras que el haber promedio tuvo un descenso de $18,5 \%$ en los últimos 4 años".

Esto demostró la paradoja de: cómo las jubilaciones caen en términos reales, a pesar de que en los últimos 15 años, el gasto público destinado al sistema previsional se multiplicó.

En ese sentido: "el nivel alcanzado por el gasto previsional en Argentina se encuentra por encima de su participación histórica en términos del producto".
${ }^{1}$ Dedicado a las autoridades de la Escuela de Economía y Negocios de la Universidad Nacional de San Martín, como también a mi mejor alumno de grado Federico Favata.

${ }^{2}$ https://www.ieral.org
EnriqueS. Dentice (h)

edentice@unsam.edu.ar

Escuela de Economía y Negocios (EEyN-UNSAM)

Director Honorario del Centro de Investigación y Medición Económica (CIME- UNSAM)

Universidad Nacional de San Martin ARGENTINA

COMO CITAR ESTE ARTÍCULO Dentice, E. S. (2021). ¿Cómo es el mapa de jubilación en Argentina?. Revista de la Facultad de Ciencias Económicas, $27(2), 25-33$.

http://dx.doi.org/10.30972/rfce.2725656

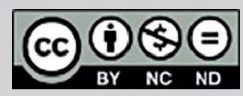

https://creativecommons org/licenses/by-ne-nd/4.0/ Revista de la Facultad de Ciencias Económicas ISSN $1668-6357$ (formato impreso) ISSN 1668-6365 (formato digital) por Facultad de Ciencias Económicas Universidad Nacional del Nordeste (UNNE) Argentina se distribuye bajo una Licencia Creative Commons Atribución - No Comercial - Sin Obra Derivada 4.0 Internacional 
"Históricamente hasta el 2005 el presupuesto previsional representaba el 5,2\% del PBI y para 2020 se había más que duplicado, al 11,9\%". "Esta dinámica se halla relacionada con las moratorias previsionales, que arrancan en 2007, y el otorgamiento de diferentes pensiones no contributivas, como la Pensión Universal al Adulto Mayor (PUAM), en vigencia desde 2017", según detalla el estudio del Instituto de Estudios sobre la Realidad Argentina y Latinoamericana (IERAL).

"El sistema jubilatorio viene incrementando su déficit año a año, y el rojo se cubre con cada vez más recursos tributarios. Precisamente, el elevado crecimiento del gasto previsional llevó el déficit previsional genuino (considerando como ingresos sólo los aportes y contribuciones a la seguridad social) de un o,5\% del PBI en 2010 a un 4,8\% en 2020".

"Una de las explicaciones del desajuste es la caída de la relación entre cantidad de trabajadores activos y beneficiarios del sistema previsional: que pasó de 2,1 en 2006 a 1,4 en 2020. La cobertura previsional está vinculada al trabajo formal pero, en la actualidad, el nivel de informalidad alcanza a la mitad de los ocupados y hay diferencias importantes por categoría ocupacional entre los aportantes, ya que los asalariados representan el 75\% y los independientes el 25\%", según el reporte.

Al mismo tiempo, también se incrementó la cantidad de beneficios previsionales que otorga el Estado. En efecto, entre el 2005 y el 2020, por ejemplo, tuvo un crecimiento de 112\% y "ante el incremento del déficit genuino del sistema, el ajuste provino finalmente para el nivel del haber jubilatorio. El haber medio cayó un 18,5\% en términos reales en los últimos cuatro años. Así, la fuerte suba en la cantidad de beneficios terminó afectando el haber de los jubilados", consideró IERAL.

El mismo centro de estudios midió que el peso de los salarios públicos y jubilaciones subió un 190 por ciento, lo que implica un peso adicional de esa partida, en relación con el PBI, de 10 puntos hasta 2017. A partir de 2018 es cuando comenzó el proceso de ajuste al sistema previsional.

Desde inicios de 2021 los haberes de los jubilados del sistema general se ajustan trimestralmente, a través de una fórmula que combina el 50\% de la variación de la recaudación de la Administración Nacional de la Seguridad Social (Anses) por beneficiario y 50\% de la variación de los salarios, según el índice del Instituto Nacional de Estadística y Censos (INDEC) o del RIPTE (Remuneración Imponible para el Trabajador Estable), el que resulte mayor.

Otro elemento a tener en cuenta en la ecuación, según IERAL, "es la cantidad de empleados públicos. La cantidad de agentes públicos en los tres niveles de gobierno (nación, provincias y municipios) pasó de: 2,2 millones en 2000 a 3,6 millones en 2020, mientras que el número de jubilados lo hizo desde: 3,3 millones a 6,8 millones en similar período. Sumando empleos públicos y jubilaciones pagadas, se pasó de 5,5 millones en el año 2000 a 10,5 millones en 2020".

"Podría decirse que se puede tener una cantidad exagerada de empleados públicos con salarios reales relativamente bajos, o una cantidad ajustada de agentes públicos con buenos salarios, pero a largo plazo resulta imposible tener un exceso de empleados estatales con salarios 
también altos. Algo similar puede decirse para el caso del sistema previsional", ha sido una de las conclusiones de la Fundación Mediterránea.

\section{¿CÓMO ES EL MAPA PARA JUBILARSE?}

El sistema jubilatorio ha tenido en los últimas años diversas modificaciones legales como normativas que dificultan el poder establecer un único método, sin dejar de mencionar que para jubilarse se debe contemplar si tal trámite se efectúa con o sin aportes completos, o con regímenes especiales de acuerdo al oficio, para autónomos y monotributistas, o prestaciones de otro tipo.

\section{EL RÉGIMEN GENERAL ORDINARIO}

Este es el primer tipo y es el que corresponde a los trabajadores en relación de dependencia, salvo a los que desempeñen trabajos que cuenten con regímenes jubilatorios especiales como es el caso de: Fuerzas Armadas; de Seguridad; Parques Nacionales; Servicio Exterior; Docentes; Judiciales; o Provincias que no hayan transferido sus cajas jubilatorias a la Nación.

El aporte, en estos casos, es mensual y se efectúa en forma de "descuentos jubilatorios" en el sueldo, contando con aportes personales equivalentes al 11\% sobre el sueldo bruto. Por su parte, el empleador debe realizar "contribuciones patronales" de: $16 \%$ calculada en base a la remuneración bruta.

\section{¿QUÉ BENEFICIOS PAGA ESTE TIPO DE JUBILACIÓN?}

Abona para quienes pasen a ser trabajadores retirados en este sistema:

- Para los varones de 65 años y mujeres de 60 -en ambos casos con 30 años de aportes comprobados que tienen derecho a una "prestación básica universal" que consta de una suma fija mensual y un $1 \%$ más adicional por cada año de servicio (que supere los 30 años de aportes, con un máximo de $15 \%$ adicional).

- Incluye "retiro por invalidez", en caso de que se acredite una incapacidad física o intelectual que implique (en términos legales) una disminución de la capacidad 
laboral igual o mayor al 66\%. Para establecerlo, éste equivale a entre 50 y $70 \%$, dependiendo de si cuenta o no con aportes del promedio de ingresos en los últimos cinco años.

- El régimen general otorga, además, una pensión por fallecimiento y una prestación por edad avanzada para mayores de 70 años con aportes de 10 años.

El total de beneficiarios de este grupo, según la Oficina de Presupuesto del Congreso (OPC), en base a datos del ANSES, arrojó que el 39,2\% cobraba la jubilación mínima. El relevamiento por sexo permite observar una mayor cantidad de mujeres en esta situación (49,3\%), casi duplicando al que comprende a los varones (26.4\%).

"Del total, 25,4\% de los beneficiarios sin moratoria, cobró la jubilación mínima. Se observó mayor cantidad de mujeres en esta situación. Esto es, $21,5 \%$ de los varones y 33\% de las mujeres beneficiarias de la Ley 24.241, sin moratorias, percibieron la jubilación mínima. Esta situación podría explicarse por las menores oportunidades en cargos y puestos con mejores salarios que suelen tener las mujeres", observó el informe del organismo que depende del parlamento.

Para quienes no llegan a completar los aportes, se abren otras maneras de poder acceder a la jubilación por la vía de distintas posibilidades de moratoria.

"Se encuentran en vigencia dos moratorias (Leyes 24.476 y 26.970), cuya población objetivo son las personas que cumplen los requisitos etarios para acceder a la jubilación, pero no poseen los años de aportes necesarios". Según OPC.

No es menor la cantidad de personas que necesitan ingresar a este tipo de "facilidades". Las mismas se cargan en las finanzas de Anses, cuando deberían estar a cargo de la Tesorería y no afectar a los incluidos en el régimen de reparto para poder jubilarse.

En marzo de 2020, fecha de corte que la OPC utilizó para su informe, se verifica que:

1. De todas las jubilaciones otorgadas por el régimen general (excluyendo especiales como docentes, investigadores o el Poder Judicial), el 55\% implicó algún tipo de moratoria.

2. Entre las mujeres, esa situación fue más común: de las 3,4 millones de moratorias habilitadas por el Estado hasta la antedicha fecha: 2,5 millones (el 73\%) correspondieron a mujeres y 819.000 a hombres.

"Puede observarse que la proporción de varones que accedieron a beneficios jubilatorios resulta prácticamente igual entre quienes accedieron mediante moratorias respecto a quienes lo hicieron sin moratoria, por lo que también se destaca una alta informalidad laboral en el sexo masculino", explicando las Moratorias para recibir la mínima. 
Los jubilados con planes especiales para completar aportes, de acuerdo con los datos al 2020, percibían mayoritariamente la jubilación mínima o, incluso, un valor menor en la retribución de bolsillo. En términos generales, los perceptores del piso de haberes se ubicaron en $87,1 \%$; agrupado por sexo: $(81,4 \%)$ en los varones y $(89,2 \%)$ mujeres.

"Por fuera del régimen general, un estudio del Centro de Implementación de Políticas Públicas para la Equidad y el Crecimiento (CIPPEC) ${ }^{3}$ clasificó a los otros sistemas ordenándolos en dos categorías:

- los regímenes diferenciales y los especiales. Los primeros son esquemas destinados a proteger a trabajadores y trabajadoras que desarrollan actividades arduas o riesgosas que podrían derivar en envejecimiento prematuro, $y$

- los segundos, son esquemas cuya especificidad se justifica en cuestiones de mérito (y que incluyen a la mayoría de los programas dirigidos a funcionarios públicos) y especificidad.

\section{MORATORIAS PARA RECIBIR LA JUBILACIÓN MÍNIMA}

Las personas jubiladas con planes especiales (para completar sus aportes), percibían la jubilación mínima o incluso menor en valores de bolsillo, en forma general; y los perceptores de este piso de haberes se ubicó en el 87,1\%, desagregado por sexo 81,4\% en los varones y, 89,2\% mujeres.

Los sistemas diferenciados son: Régimen Simplificado de Impuestos y ocupados en casas particulares. "El régimen de monotributo es una variación del sistema de trabajo autónomo que permite, en su dimensión previsional, a trabajadores independientes hacer aportes en relación a un ingreso supuesto, que es extremadamente bajo" según CIPPEC.

"Esto implica que su beneficio eventual tiene un fuerte componente de subsidio, dada la aplicación del haber mínimo: el aporte promedio del régimen equivalía, en 2020, a un 4\% del haber inicial de las altas otorgadas dicho año, mientras que en el régimen general este porcentaje era del $26 \%$. Entre 2018 y 2020, cerca de $32 \%$ de los nuevos jubilados accedieron a su beneficio bajo este régimen", en base al informe CIPPEC.

"Por último, el régimen para el empleo en casas particulares consiste en un esquema tarifado (es decir que los aportes no son proporcionales al salario) y donde el subsidio es casi total, en tanto el aporte mensual equivale a un 0,5\% del haber de alta. Este régimen explica cerca de un $7,5 \%$ del total de altas jubilatorias", continuó CIPPEC. 


\section{SISTEMAS DIFERENCIALES}

El conglomerado de sistemas diferenciales es muy amplio. "En 2021 existen y conviven al menos 38 regímenes diferenciales, cubriendo a grupos de trabajadores/as tan diversos como quienes realizan tareas en minas subterráneas o bailarines/as y cantantes líricos/as del Teatro Colón", mencionó el trabajo de ese centro de estudios.

Este tipo de regímenes permite reducciones en las edades mínimas para la jubilación de 5 o 10 años, en relación con el esquema general.

Entre esos oficios, se encuentran aquellos que implican:

- trato o contacto directo con los pacientes de enfermedades infectocontagiosas o mentales,

- en cámaras frías o en tareas declaradas insalubres,

- actividades ferroviarias,

- conducción de ómnibus o vehículos de transporte colectivo de personas,

- tareas mineras a cielo abierto,

- tareas de laminación, acería y fundición,

- aeronavegación,

- en la Antártida e Islas del Atlántico Sud,

- personal embarcado,

- de la industria del vidrio,

- de la industria del chacinado y de la carne.

- estibadores portuarios,

- capataces y güincheros,

- policía de establecimientos navales,

- operadores de telegrafía y radiotelegrafía,

- personal de seguridad operativa industrial,

- señaleros ferroviarios,

- taxistas autónomos,

- servicios eléctricos,

- petroleros,

- ballet estable y contratado y cantantes líricos del Teatro Colón,

- enfermería

- docentes de escuelas de zonas y áreas de frontera, entre otros. 


\section{REGÍMENES Y GÉNERO}

Regímenes diferenciales para:

- el personal femenino de empresas telefónicas,

\section{¿CUÁNTOS REGÍMENES ESPECIALES EXISTEN?}

Respecto a los regímenes especiales existen siete, administrados por ANSES:

- docencia no universitaria;

- docencia universitaria nacional;

- investigación científica y técnica;

- funcionarios/as del Poder Judicial y del Servicio Exterior

- trabajadores/as del sindicato Luz y Fuerza y

- trabajadores/as de Yacimientos Carboníferos de Rio Turbio.

Por su naturaleza, estos sistemas poseen haberes promedio mayores al régimen general.

Encabezados por el del Servicio Exterior y el del Poder Judicial, donde se concentran la mayor cantidad de beneficios, por encima del valor de dos jubilaciones máximas.

La mayor parte de los docentes no universitarios perciben entre dos y cuatro haberes mínimos.

Los docentes universitarios lo hacen en un rango amplio de entre dos jubilaciones mínimas y dos máximas.

Entre los investigadores, sus haberes suman en la mayoría de los casos entre una y dos jubilaciones máximas. Entre los trabajadores retirados de Luz y Fuerza, la mayor parte de las mujeres cobran un haber que equivale a entre dos y cuatro haberes mínimos, y entre cuatro mínimas y una máxima para los hombres.

Los empleados en Yacimientos Carboníferos de Río Turbio cobran, tras su retiro, una jubilación equivalente a entre una y dos jubilaciones máximas para los hombres en la mayoría de los casos, mientras que la mitad de mujeres perciben entre 4 mínimas y una máxima.

Por fuera del esquema jubilatorio general, de las moratorias y de los regímenes diferenciados, existe otro entramado de sistemas previsionales que incluye "el personal de las fuerzas armadas y de seguridad, de empleados públicos provinciales y municipales y las pensiones no contributivas".

En este último grupo, además de las pensiones por: 1) invalidez, 2) vejez y las 3) de madres de siete hijos o más, se encuentra la Pensión Universal para el Adulto Mayor (PUAM), creada en 2016 para personas que no están alcanzadas por ninguna jubilación y que equivale al $80 \%$ de un haber mínimo. 
También existen cajas particulares para distintas profesiones, como abogados, bioquímicos, profesionales en ciencias económicas, escribanos, farmacéuticos, martilleros, médicos, agrimensores, arquitectos, ingenieros, odontólogos, kinesiólogos, veterinarios, psicólogos, entre otros, que poseen un régimen peculiar y diferenciado.

Consideramos que la cantidad de normativa diversa y dispersa sobre el sistema previsional hace que sea imposible alcanzar la seguridad jurídica en materia de jubilaciones. Por lo demás, también influye en ello que la necesidad económica planteada por la crisis es de tal magnitud, que los Gobiernos toman el dinero de la seguridad social para financiar otros rubros, lo que conspira contra la confiabilidad del sistema y obliga a permanentes cambios, en desmedro de la certeza acerca de los regímenes jubilatorios, principalmente, a futuro.

\section{BIBLIOGRAFÍA CONSULTADA}

Análisis de Sustentabilidad del Sistema Previsional Argentino Proyecciones 2015 2019. Dirección de Relaciones Fiscales. Gobierno de la Ciudad Autónoma de Bs.As., junio 2015. Disponible en: https://nanopdf.com/download/sustentabilidad-del-sistema-previsional-argentino_pdf

El sistema Previsional en la Argentina, CIPPEC 2020.

Disponible en: https://www.cippec.org/proyecto/el-sistema-previsional-en-argentina/

Jubilación y movilidad jubilatoria. Resultados 2020 y escenarios 2021. IERAL. Disponible en: https://www.ieral.org/noticias/jubilaciones-movilidad-escenarios-para-2021-4322.html

Jubilaciones: cuáles son los regímenes vigentes en la Argentina y cuánto se cobra en cada uno. Infobae, 25.07.21. Disponible en: https://www.infobae.com/economia/2021/07/25/jubilacionesuno-por-uno-cuales-son-los-regimenes-vigentes-en-la-argentina-y-cuanto-se-cobra-en-cada-uno/

Régimen Previsional Público. Unificación. Ley 26425. Disponible en: http://servicios.infoleg.gob.ar/infolegInternet/anexos/145000-149999/148141/norma.htm

Sistema Integrado de Jubilaciones y Pensiones. Ley 24241.

Disponible en: http://servicios.infoleg.gob.ar/infolegInternet/anexos/o-4999/639/texact.htm

Sistema Previsional Argentino - Evolución, Proyección y Reforma. Fundación Konrad Adenauer Stiftung, Bs.As. Disponible en: https://www.kas.de/documents/287460/4262432/sistema_ previsional_FINAL.pdf/1fo4acbe-a19d-d45d-1636-fo86c9738ff3?t=1610636786393 


\section{CURRICULUM VITAE}

\section{Enrique S. Dentice (h)}

Dieciocho años de investigación empírica de la realidad económica.

Desde el año 2000 me desempeño como Coordinador del CIME (Centro de Investigación y Medición Económica de la EEyN-UNSAM), generando informes de carácter macroeconómico, proyecciones de variables económicas, tanto para las publicaciones propias de la universidad, presentación en congresos internacionales, regionales y nacionales, como también a nivel empresario. Participación activa en el relevamiento de expectativas de mercado del BCRA. Complementado por una labor docente, que resulta ser la receptora de los estudios realizados. Anteriormente me desempeñé en el sector privado en puestos relevantes en el sector financiero de empresas extranjeras. Asimismo he llevado adelante asesoramientos al estado nacional en materia de deuda externa como en conjunto con el Instituto Universitario de Seguridad Marítima en el tema de hidrovía.

edentice@unsam.edu.ar 